\title{
Repetition effects with kinesthetic and visual-kinesthetic stimuli
}

\author{
BETTY ANN M. TURPIN \\ National Research Council of Canada, Ottawa, Ontario, Canada \\ and \\ GEORGE E. STELMACH \\ University of Wisconsin, Madison, Wisconsin
}

\begin{abstract}
Research in choice reaction time typically has revealed that reaction time is faster for repeated events than for nonrepeated events. The two experiments reported examined the characteristics of the repetition effect when two successively presented stimuli were either both kinesthetic or both visual-kinesthetic. A stimulus-priming paradigm in which the prime-target stimulus sequence was either a repetition or a nonrepetition of movement-direction information was employed. The data revealed that the typical repetition effect occurred within and between modalities, with repetitions yielding faster reaction times than nonrepetitions, and that the interstimulus time intervals did not alter the benefit of a repetition.
\end{abstract}

One particular information-processing phenomenon that has been intensely scrutinized over the years is the "repetition effect" (Bertelson, 1961; Hyman, 1953). This robust effect is characterized by the occurrence of faster reaction times to repeated events than to nonrepeated events (e.g., Bertelson, 1961, 1963; Bertelson \& Renkin, 1966; Kornblum, 1973; Rabbitt, 1968; Remington, 1969). However, evidence is equivocal with respect to whether the repetition effect is caused by repeated stimuli (Bertelson \& Tisseyre, 1966; Smith, 1968), activated response codes (Bertelson, 1965; Rabbitt, 1968), or a combination of both. Since the magnitude of the repetition effect is a function of the interstimulus interval (ISI), Hyman (1953) and Kornblum (1973) have speculated that the repetition effect may be partially attributed to memory-dependent operations. The most prominent explanation of the repetition effect was proposed by Bertelson (1963), who suggested that the advantage of a repetition was a function of bypassing the memory-retrieval stage. Bertelson proposed that, when a subsequent stimulus is the same as the previous stimulus, then a memory search, to identify the stimulus, is not necessary and the previously retrieved identical response is executed.

Typical paradigms employed to examine the repetition effect have required subjects to respond to one stimulus event at a time wherein the existing relationships have varied between the stimulus and the response. The most common methodologies employed have manipulated the number of alternatives and have imposed a

B. A. M. Turpin's mailing address is: Division of Mechanical Engineering, National Research Council of Canada, Ottawa, Ontario, Canada K1A 0R6. G. E. Stelmach's mailing address is: Motor Behavior Laboratory, University of Wisconsin, Madison, Wisconsin 53706. probability assignment to the occurrence of stimuli. In addition, in conjunction with varying the S-R compatibility, several experiments have used stimulus-response mappings that comprised one-to-one or many-to-one mappings. The results of these studies indicate that increasing the number of possible events or the stimulusresponse compatibilities increases the size of the repetition effect (Bertelson, 1963; Hyman, 1953; Keele, 1969). Furthermore, these various manipulations have been employed in serial or choice reaction time tasks and have produced similar results (see Kornblum, 1973, for a review).

The repetition effect also has been shown to dissipate as the ISI increases. Bertelson (1961), Bertelson and Renkin (1966), and Hale (1967) found the repetition effect to decrease as the interval increased from $100 \mathrm{msec}$ to $2 \mathrm{sec}$ for repeated events, and Smith (1968) found similar results with intervals ranging from 2 to $10 \mathrm{sec}$. However, Keele (1969) found no repetition effect for compatible tasks with intervals of 2,4 , and $8 \mathrm{sec}$, but he did find the effect for incompatible tasks, as did Rabbitt (1968) and Schvaneveldt and Chase (1969). Furthermore, with intervals longer than $300 \mathrm{msec}$, there is a tendency for nonrepetitions to be as fast as repetitions (Entus \& Bindra, 1970; Williams, 1966).

The experiments reported here focus on the relationship between stimulus repetitions (a prime-target sequence) and their ISI. The primary purpose of the study was to determine whether the repetition effect is characteristic of processing within and between the visual and kinesthetic modalities, and whether this effect is differentially influenced by the ISI. To assess the characteristics of the repetition effect under sensory modality manipulations, the experiments adopted a modified stimulus priming technique (Beller, 1971), wherein 
the initial stimulus (prime) presented did not convey direct information pertaining to the subsequent stimulus (target). Repetitions occurred between two successively presented stimuli and the response required was kept constant.

\section{METHOD}

Subjects. Twelve task-naive, right-handed (male and female) subjects recruited from the University of Wisconsin-Madison participated in the two experiments. Each experiment involved six subjects and was distinguishable by the variation of the kinesthetic-kinesthetic $(\mathrm{K}-\mathrm{K})$ and visual-kinesthetic (V-K) stimulus sequence.

Apparatus. A finger actuator, which consisted of a finger cylinder with a displacement range of $.635 \mathrm{~cm}$ in three directions (up, right, left), was mounted in a vertical panel and used for the presentation of direction cues. Springs from three solenoids were affixed to the outer edge of the finger cylinder such that, when the solenoids were activated, the solenoid pulled the finger cylinder $.5 \mathrm{~cm}$. The three movement solenoids were encased in a vertical panel, and extending from the base of the vertical panel was a horizontal platform, the height of which could be adjusted, that served as a hand/arm rest for subjects while the index finger rested in the cylinder. Located in the upper right-hand corner of the vertical panel was a red LED that functioned as a warning light. A visual display consisting of three equidistantly separated arrows was attached to the top of the vertical panel. Five-volt LEDs, one below each arrow, could be illuminated independently and served to provide visual direction information.

Procedure. The subjects participated in four sessions: The 1st day involved a familiarization period, and the remaining 3 were test days. The prime-target sequence varied the modalities involved in each experiment. For example, in the V-K experiment, the prime was visual and the target was kinesthetic. Prime-target sequences consisted of directional information denoted as rightright, left-left, left-right, and right-left. The subjects were required to report verbally the direction of the target (second stimulus) as quickly and as accurately as possible. After each trial, the subjects were given reaction time feedback and were informed of the correctness of the response.

On a typical trial, $1 \mathrm{sec}$ after a warning signal, the primetarget sequence was presented with variable ISIs $(50,150$, or $300 \mathrm{msec}$ ). The reaction time of the subject's verbal response to the second of two stimuli was detected by a unidirectional boom-type microphone and recorded by a PDP-8e computer via a noise-operated relay (Hunter Model 3205). The experimental setup was designed such that the reaction time clock was initiated with the presentation of the second stimulus and was terminated by the subject's verbal response. The intertrial interval was approximately 4 sec.

Design. In each modality experiment, the following independent variables were manipulated: prime-target sequence, ISIs, and days. On Day 1, the subjects received 216 trials ( 3 blocks), and on Days 2 and 3 the subjects received 288 trials ( 4 blocks/day). Within a block of trials, the subjects received randomized trials from among the four prime-target sequences, with six trials given at each prime-target and ISI combination. Reaction times for each experiment were analyzed in a subjects (6) x primetarget (2) x ISI (3) within-subject design.

\section{RESULTS}

Since the methodological and analytical procedures were identical for both the K-K and V-K experiments, the results of these experiments will be presented con-

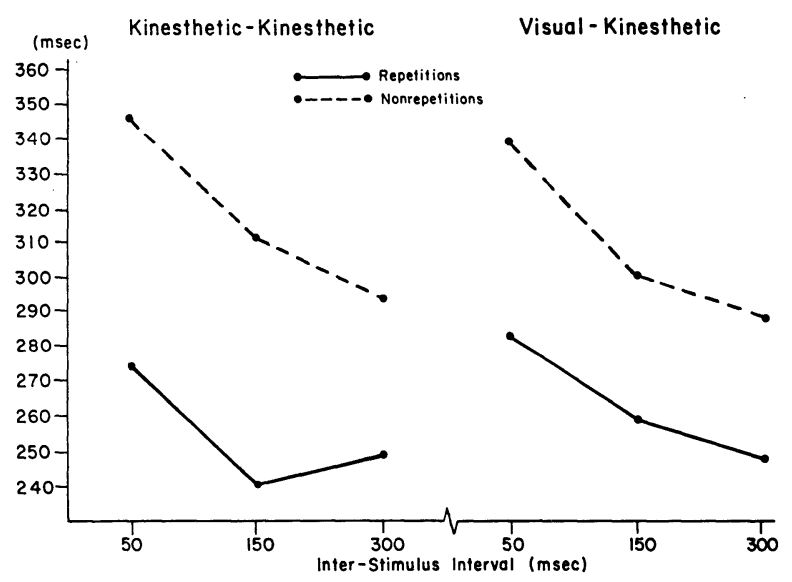

Figure 1. Mean reaction time for repetitions and nonrepetitions plotted as a function of ISIs for the K-K and the V-K experiments.

currently. To determine the characteristics of the repetition effect for each prime-target sequence, analyses were performed separately for each modality experiment.

The mean reaction times for repetitions and nonrepetitions as a function of the ISI for the K-K and V-K experiments are plotted in Figure 1. As can be clearly seen, there are prominent repetition and ISI effects. Reaction times for repetitions are significantly faster than those for nonrepetitions by $62 \mathrm{msec}$ $[F(1,90)=77.13, p<.001]$ in the K-K experiment and by $46 \mathrm{msec}[F(1,90)=15.02, p<.025]$ in the V-K experiment. The reaction times also decreased as a function of ISI in both K-K and V-K experiments $[\mathrm{Fs}(2,90)=$ $11.65, \mathrm{p}<.025$, and $4.86, \mathrm{p}<.025$, respectively]. The repetition $x$ ISI interactions were nonsignificant in each experiment, indicating that ISI did not differentially affect the occurrence of the repetition effect.

\section{DISCUSSION}

There were two major aspects of the repetition effect that were under scrutiny in these experiments: (1) the assessment of the robustness of the repetition effect as influenced by stimulus modalities (intra- and intermodality effects), and (2) the assessment of the ISI on the repetition effect for each stimulus modality manipulation. The data clearly indicated that the stimulus repetition effect did occur under both manipulations. Although subjects had no reason to attend to the first stimulus, since no expectancy of the second stimulus existed as a function of the direction of the first stimulus, faster processing was achieved when the prime $\left(S_{1}\right)$ and target $\left(S_{2}\right)$ were identical. This outcome typifies the repetition phenomenon that has promoted extensive study over the last 20 years (Bernstein, 1970; Bernstein \& Clark, 1971; Bertelson, 1963, 1965). Moreover, the finding that the repetition effect is not limited to intrasensory presentations suggests that the repetition effect is not caused by specific pathway activation and that it may be vested in high-level mental operations that likely involve memory mechanisms. Along these lines, Dyer (1973) and Keele (1973), who have observed that subjects have difficulty in ignoring information that they otherwise have been told to disregard or that is irrelevant, have speculated that stimuli may activate response codes in memory regardless of whether those stimuli convey relevant information. 
There is no way of knowing whether the subjects "used" the first stimulus when presented, thereby initiating such processes as encoding and retrieval. Consequently, if covert processes occurred when the stimuli were redundant (repetition), most of the early processing would be completed and the subject primarily had only to execute an already retrieved response code. The data revealed that the magnitude of the repetition effect was not differentially affected by the ISI but that reaction time did decrease as the ISI increased. This is not the typical repetition effect; most studies have reported that the magnitude of the repetition effect dissipates after $150 \mathrm{msec}$. There was some suggestion of a reduction in the repetition effect in the K-K experiment at the $300-\mathrm{msec}$ ISI, but none in the V-K experiment. Why there is a slight difference between experiments is not clear. However, it may be partially explained by the speed of processing within and between modality experiments. The reaction times for the nonrepetition conditions in both the K-K and V-K experiments are practically identical. However, the reaction times observed for the repetition condition in the K-K experiment are slightly faster than those in the V-K experiment at the three ISIs. This suggests that there is greater benefit of processing when the successive stimuli presented are in the same modality. This faster processing may have made this experiment more sensitive to retrieval processes, as indicated by the fact that the repetition effect was optimal at $150 \mathrm{msec}$.

Inspection of Figure 1 reveals that the ISI did not differentially affect the reaction times for repetitions and nonrepetitions, except, perhaps, at the $300-\mathrm{msec}$ ISI in the K-K experiment. Overall, for both experiments, processing was most efficacious at the $300-\mathrm{msec}$ ISI and was shown to dissipate as the ISI decreased. This finding is in agreement with the data from Posner and Snyder's (1975a, 1975b) studies, in which they found that the maximum benefit in processing rate occurred at ISIs of around $300 \mathrm{msec}$.

In conclusion, these data have demonstrated that the repetition effect occurs within and between modalities. This finding alone suggests that the increase in speed associated with responding to successively repeated stimuli is localized in higher level cognitive processing and is not dependent upon specific sensory biases. The exact type of mental operations involved has not been determined, but it remains possible that the presentation of the first stimulus may activate encoding and selection processes such that, when the stimulus is repeated, the required response is immediately available for execution (Shiffrin \& Schneider, 1977). That there was little change in the magnitude of the repetition effect across the three ISIs seems to support this type of interpretation.

\section{REFERENCES}

Beller, H. K. (1971). Priming: Effects of advance information on matching. Journal of Experimental Psychology, 87, 176-182.

Bernstein, I. H. (1970). Can we see and hear at the same time? Some recent studies of intersensory facilitation of reaction time. In A. F. Sandus (Ed.), Attention and performance III (pp. 36-51). Amsterdam: North-Holland.

Bernstein, I. H., \& Clark, M. H. (1971). Intersensory effects in the psychological refractory period. Perception \& Psychophysics, 9, 135-139.

Bertelson, P. (1961). Sequential redundancy and speed in a serial two-choice responding task. Quarterly Journal of Experimental Psychology, 12, 90-102.

Bertelson, P. (1963). S-R relationships and reaction times to new versus repeated signals in a serial task. Journal of Experimental Psychology, 65, 478-484.

Bertelson, P. (1965). Serial choice reaction time as a function of response versus signal-and-response repetition. Nature, 206, 217-218.

Bertelson, P., \& Renkin, A. (1966). Reaction time to a new versus repeated signals in a serial task as a function of responsesignal time interval. Acta Psychologica, 25, 132-136.

Bertelson, P., \& Tisseyre, F. (1966). Choice reaction time as a function of stimulus versus response relative frequency of occurrence. Nature, 212, 1069-1070.

DYER, F. N. (1973). The Stroop phenomenon and its use in the study of perceptual, cognitive, and response processes. Memory \& Cognition, 1, 106-120.

Entus, A., \& Bindra, D. (1970). Common features of the "repetition" and "same-different" effects in reaction time experiments. Perception \& Psychophysics, 7, 143-148.

HaLe, D. J. (1967). Sequential effects in a two choice serial reaction task. Quarterly Journal of Experimental Psychology, 78, 133-141.

Hyman, R. (1953). Stimulus information as a determinant of reaction time. Journal of Experimental Psychology, 45, 188-196.

Keele, S. W. (1969). Repetition effect: A memory dependent process. Journal of Experimental Psychology, 80, 243-248.

Keele, S. W. (1973). Attention and human performance. Pacific Palisades, CA: Goodyear.

Kornblum, S. (1973). Sequential effects in choice reaction time: A tutorial review. In S. Kornblum (Ed.), Attention and performance IV (pp. 259-288). Amsterdam: North-Holland.

Posner, M. I., \& SNyder, C. R. R. (1975a). Attention and cognitive control. In $\mathrm{R}$. L. Solso (Ed.), Information processing and cognition (pp. 55-85). Hillsdale, NJ: Erlbaum.

Posner, M. I., \& Snyder, C. R. R. (1975b). Facilitation and inhibition in the processing of signals. In P. M. A. Rabbitt (Ed.), Attention and performance $V$. London: Academic Press.

RABbitT, P. M. A. (1968). Repetition effects and signal classification strategies in serial choice-response tasks. Quarterly Journal of Experimental Psychology, 20, 232-240.

Remington, R. J. (1969). Analysis of sequential effects in choice reaction times. Journal of Experimental Psychology, 82, 250-257.

Schvaneveldt, R. W., \& Chase, W. G. (1969). Sequential effects in choice reaction time. Journal of Experimental Psychology, 80, 1-8.

Shiffrin, R. M., \& Schneider, W. (1977). Controlled and automatic human information processing: II. Perceptual learning, automatic attending, and general theory. Psychological Review, 84, 127-190.

Sмiтн, M. C. (1968). Repetition effect and short-term memory. Journal of Experimental Psychology, 77, 435-439.

Williams, J. A. (1966). Sequential effects in disjunctive reaction time: Implications for decision models. Journal of Experimental Psychology, 71, 655-672.

(Manuscript received for publication November 15, 1983.) 